\title{
Una huella del academicismo arquitectónico en Córdoba: la torre de la iglesia de Santa María Magdalena
}

\author{
Jesús María Ruiz Carrasco \\ Universidad de Córdoba \\ L92rucaj@uco.es
}

RESUMEN: La llegada del prelado Antonio Caballero y Góngora a la Diócesis de Córdoba significó la puesta en marcha de un proceso destinado a implantar a lo largo y ancho de la misma los novedosos preceptos artísticos academicistas. Para procurar su éxito requirió el establecimiento en Córdoba de tres artistas académicos, entre los que se encontraba Ignacio Tomás, arquitecto encargado de la proyección y dirección de nuevas obras constructivas que simbolizaran públicamente el codiciado cambio estético. Ejemplo de su actividad en la ciudad cordobesa es la torre de la iglesia de la Magdalena, cuya edificación representa cada una de las circunstancias que condicionaron la consecución de la iniciativa episcopal. Una obra paradigmática de la introducción del academicismo arquitectónico en Córdoba.

PALABRAS CLAVE: Córdoba; llustración; Academia; Arquitectura; Ignacio Tomás.

\section{A Trace of Architectural Academicism in Córdoba: the Tower of the Church of Santa María Magdalena}

ABSTRACT: The arrival of the prelate Antonio Caballero y Góngora to the Diocese of Córdoba brought about the implementation of a process aimed at implanting the new artistic precepts of academicism throughout the length and breadth of the diocese. To ensure its success, it required establishing three academic artists in Córdoba, including lgnacio Tomás, architect in charge of projecting and directing new construction works that publicly symbolized the coveted aesthetic change. An example of his activity in the city of Cordoba is the tower of the Magdalena church, where the construction represents the circumstances that conditioned the achievement of the episcopal initiative. It is a paradigmatic work of the introduction of architectural academicism in Córdoba.

KEYWORDS: Córdoba; Enlightenment; Academy; Architecture; Ignacio Tomás.

Recibido: 28 de febrero de 2020 / Aceptado: 7 de junio de 2020.

Córdoba, «patria» que había sido de «profesores muy acreditados», se hallaba en la década de 1780 «enteramente destituida de medios y de estímulos que resucitasen en ella la antigua inclinación a las artes del diseño", ya que «las obras públicas, la decoración de los templos y todo lo demás» fueron emprendidas o continuadas entonces «sin verdaderas máximas ni principios fundados» (Real Academia de San Fernando, 1790: 5-6). Con estas palabras la Real Academia de San Fernando denunció en 1790 la ausencia de promotores y artistas capaces de implantar hasta aquel momento en Córdoba los preceptos estéticos de raigambre clásica y fundamentos ilustrados fomentados por sus miembros ${ }^{1}$, tal y como aconteció paralelamente en otras regiones del Reino (Bédat, 1989: 378-383; García, 1991: 339-341). Sin embargo, el contexto artístico cordobés experimentó justamente a partir de la fecha referida un cambio sustancial, como consecuencia de un suceso de especial significación: la llegada del nuevo prelado de su Diócesis, Antonio Caballero y Góngora².

La presencia de Caballero y Góngora, destacada figura eclesiástica de la segunda mitad del siglo XVIII en el ámbito hispánico, anteriormente virrey y capitán general de Nueva Granada, Gran Cruz de la Distinguida y Real Orden de Carlos III,

Cómo citar este artículo: RUIZ CARRASCO, Jesús María, "Una huella del academicismo arquitectónico en Córdoba: la torre de la iglesia de Santa María Magdalena", Boletín de Arte-UMA, n. ${ }^{\circ}$ 42, Departamento de Historia del Arte, Universidad de Málaga, 2021, pp. 165-174, ISSN: 0211-8483, e-ISSN: 2695-415X, DOI: http://dx.doi. org/10.24310/BoLArte.2021.vi42.8143 
dedicado coleccionista, reconocido erudito y personalidad comprometida con el movimiento cultural ilustrado (Pérez, 1951), favoreció la implantación de los preceptos artísticos academicistas en Córdoba. Como punto de partida para iniciar dicho proceso introductorio, impulsó la creación de una Escuela de Dibujo (Rey, 1923: 57-62; Aranda, 1986) donde, bajo su patronazgo, debía instruirse a los futuros artistas siguiendo el modelo de la Academia madrileña, la cual calificó el proyecto cordobés como el «medio» para recobrar «las luces perdidas» (Real Academia de San Fernando, 1790: 6). Con el fin de poner en marcha la iniciativa, Caballero precisó del establecimiento en Córdoba de los académicos Ignacio Tomás, Joaquín Arali y Francisco Agustín ${ }^{3}$, que vinieron no solo para dirigir como docentes las secciones de Arquitectura, Escultura y Pintura de la nueva escuela respectivamente, sino también para trabajar en diferentes empresas artísticas al servicio del Prelado (Ponz, 1792: 37-38). Concretamente, Ignacio Tomás (Sambricio, 1986: 423-430; Serra, 2014), que se trasladó a Córdoba en febrero de $1790^{4}$, fue nombrado ese mismo año responsable de las obras parroquiales de la Diócesis cordobesa, "arquitecto de cámara» de su obispo ${ }^{5}$ y, en 1791, maestro mayor de obras de su Catedral ${ }^{6}$. A partir de entonces dirigió y planteó varios proyectos constructivos en las diferentes parroquias de la Diócesis, entre los que destacó la edificación de una nueva torre para la, entonces, parroquia de Santa María Magdalena de Córdoba.

\section{Elaboración y puesta en marcha de un proyecto academicista}

La actualmente desacralizada iglesia de Santa María Magdalena ${ }^{7}$ [1], de fundación medieval ${ }^{8}$, fue objeto de varias reformas estructurales a lo largo del siglo XVIII, cuando también se le añadieron la mayoría de los desaparecidos retablos y ornatos que decoraban su interior (Ramírez de Arellano, 1873: 9-22; Raya, 1987: 65, 67, 94, 96, 194). Fue durante el transcurso de las obras efectuadas en la capilla de la Virgen de los Dolores, en enero de 1791, cuando el «maestro de arte de albañilería» Mariano Luque detectó que la torre de la parroquia amenazaba ruina "por dos partes", lo cual no solo conminaba su solidez, sino también la de la capilla mayor. Esta situación llevó a José del Castillo y Valle, rector de la iglesia, a solicitar a la prelatura cordobesa una solución al respecto el 31 de enero. En consecuencia, el 13 de febrero, la Mitra comisionó a Ignacio Tomás para que reconociera el estado de la fábrica parroquial. Una vez efectuada dicha inspección, el arquitecto académico advirtió en un informe firmado el 20 de febrero que la base de la torre se hallaba desplomada hacia la «parte exterior y calle», lo cual causaba quiebras en la pared que la unía con la de la capilla mayor. Asimismo, señaló que, si bien la única solución posible era la de engatillar dichos muros con tirantes de hierro ${ }^{9}$, la intervención entrañaba un riesgo difícilmente asumible, dado que las fuerzas ejercidas por el continuo desplomo y el «vaivén» de la torre podrían provocar la futura ruina de la cabecera del edificio. Por todo ello Tomás concluyó que era conveniente la construcción de una nueva torre, a pesar del gasto que conllevaría. El dictamen fue ratificado por la autoridad episcopal el 28 de febrero ${ }^{10}$.

El propio Ignacio Tomás fue el encargado de elaborar el proyecto constructivo, el cual, entregado el 2 de julio, estaba formado por un diseño al que acompañaba un documento donde se especificaban las siete "condiciones" que se debían observar para la satisfactoria culminación de la obra ${ }^{11}$. A pesar de que no se tiene constancia de la traza, sí se ha conservado el escrito explicativo adjunto a la misma; cuyo contenido, junto a los datos sobre el proceso constructivo de la torre y a la morfología de la misma una vez finalizada, ilustran el proyecto ideado por Tomás.

El arquitecto académico inició la exposición de las referidas «condiciones» desarrollando las medidas, la composición y el método para llevar a cabo la cimentación de la obra. En concreto, determinó que los cimientos debían tener 12 pies $(3,34 \mathrm{~m})$ de profundidad y exceder en una vara (83 $\mathrm{cm}$ ) el ancho del alzado de la torre en aquellas partes donde la antigua fábrica lo permitiera; estar compuestos de «hormigón macizo» de guijo "gordo y menudo", cuya mixtura sería de dos partes compuestas por "espuertas de granza» extraídas del «hundimiento» de la anterior torre, otras dos partes de cal y una de arena ${ }^{12}$; y ser dispuestos a base de tongadas de una cuarta $(21 \mathrm{~cm})$ asentadas con "pisón agudo", dando lugar a una masa «algo dura» sobre la que superponer alternadamente más guijo «a golpe de pisón pesado y llano», quedando así «uno con otro bien incorporado y macizo» hasta culminar la cimentación ${ }^{13}$.

Con respecto al alzado de la torre, Tomás expuso su parecer en los puntos segundo y tercero del informe. Por un 

1. Exterior de la iglesia de Santa
María Magdalena de Córdoba

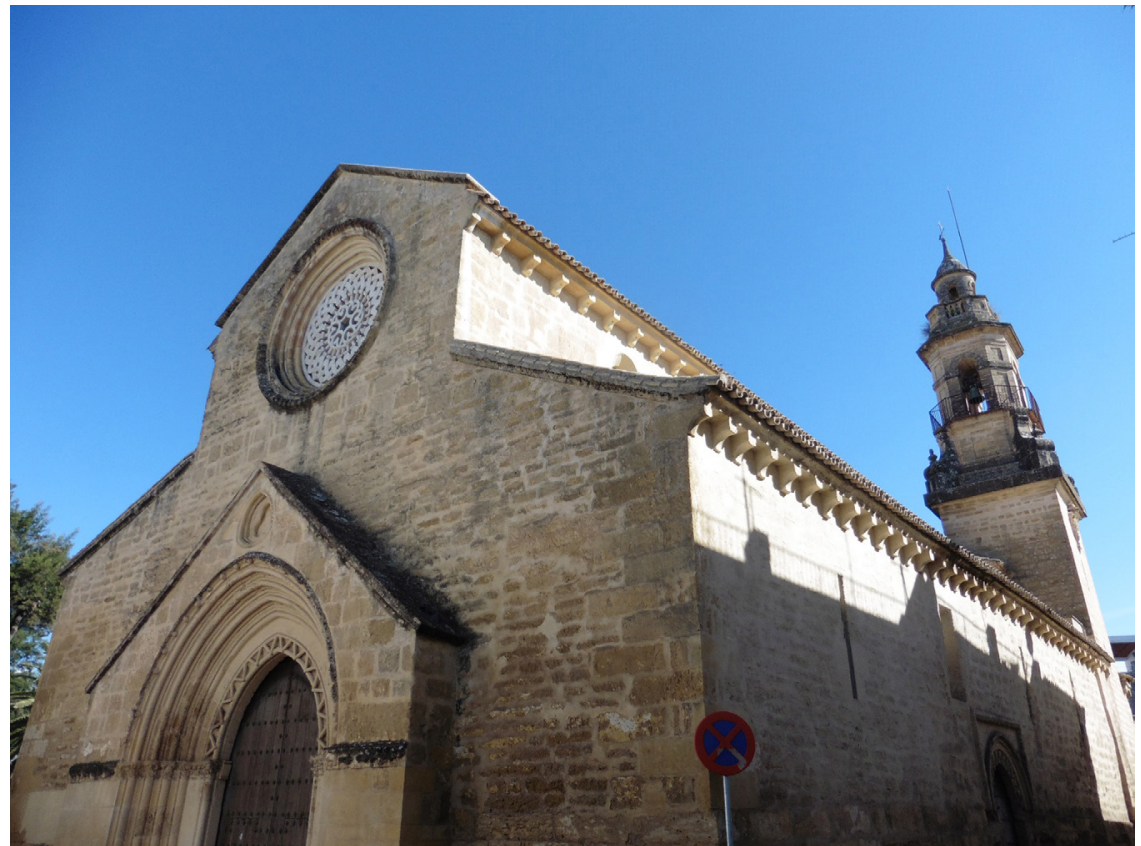

lado, dispuso que el primer cuerpo, cuya elevación alcanzaría la del alero principal de la iglesia, estaría apoyado sobre un zócalo igual de alto que el de la construcción medieval y conformado por cantería labrada de tizones interpolados de no más de una vara $(83 \mathrm{~cm})$ de ancho; que para completar lo que restaba de la construcción hasta la primera imposta se utilizaría mampostería procedente de la antigua torre, cuyos paramentos se labrarían y arreglarían para formar «buena cara en lo exterior» ${ }^{14}$; así como que todo ello se llevaría a cabo a la vez que la escalera helicoidal del interior, compuesta por peldaños de piedra labrados y un «tabique de ladrillo» que formaría el «ojo» destinado al toque de campanas, e iluminada por «lumbreras». Por el otro, determinó la inclusión de un gotero ${ }^{15}$ de 3 pies y medio $(97 \mathrm{~cm})$ de ancho en cada una de las tres impostas de la torre, debiendo ser éstas, el segundo cuerpo, arcos y dinteles del tercero, pedestales, pasamanos, balaustres y remate de piedra; en contraposición a las "primeras gradas», el sotabanco del cuerpo de campanas, las bóvedas y los antepechos superiores, que serían de ladrillo. Por último, advirtió que la fábrica parroquial debía costear la colocación sobre las «ventanas de campanas» de un cincho de hierro que engrillara la zona más alta de la torre, la introducción de un nabo de hierro dentro del remate para superponer tanto una cruz como una veleta del mismo material, y el emplazamiento de las propias campanas ${ }^{16}$.

En los cuatro apartados restantes Tomás expuso las obligaciones del asentista encargado de la edificación y el costo de la misma. En primer lugar, advirtió que la obra debía ejecutarse conforme al proyecto presentado, pudiendo este ser modificado durante su ejecución siempre que fuese oportuno. Seguidamente, puntualizó que la fábrica debería ir trabajada con la mayor solidez y que los materiales empleados serían de la mejor calidad. Además, en el sexto apartado precisó que era imprescindible macizar las ventanas y las hendiduras de la cabecera de la iglesia, «acompañar los tejados» hasta unirlos con la nueva torre y limpiar los escombros producidos por la obra. Por último, detalló la forma de sufragar los materiales y los jornales destinados a la ejecución del proyecto, cuyo coste total estimó en 56.860 reales de vellón ${ }^{17}$.

Dos días después de la entrega del proyecto, el 4 de julio, la Mitra solicitó información de los caudales disponibles al tesorero del Obispado, Juan Ramón Gómez Mayoral, quien esa misma jornada confirmó la existencia de medios suficientes para emprender la obra. De este modo, justo al día siguiente, el gobernador, provisor y vicario general de la ciudad de Córdoba, Diego Antonio Navarro Villodres ${ }^{18}$, ordenó el ini- 
cio de las obras, cuya ejecución seguiría el proyecto de Tomás y correría a cargo del maestro alarife Mariano Luque, que dispuso de 60.000 reales de vellón para edificar la torre ${ }^{19}$.

El proceso edificatorio se desarrolló inicialmente con celeridad, pues el día 2 de noviembre la obra se encontraba aproximadamente a la mitad de su ejecución. Fue en esta misma fecha cuando, por indicación de Ignacio Tomás, se encargó a Manuel Sánchez Sandoval la realización del escudo de armas del obispo Caballero y Góngora que se iba a colocar en el cuerpo principal de la torre [2]. El blasón pétreo fue concluido y entregado el 4 de abril de 1792, ascendiendo su coste hasta los 800 reales de vellón ${ }^{20}$. Este dato puede resultar sorprendente, ya que, si bien Tomás indicó la conveniencia de designar a Sánchez Sandoval como artífice de la obra del escudo tras examinar el diseño que había realizado y a pesar de las limitadas posibilidades decorativas que ofrecía la realización de una representación heráldica en piedra, la producción anterior del mencionado tracista y escultor cordobés se caracterizó por la profusión ornamental de sus obras ${ }^{21}$. Esta última circunstancia supondría en principio una diferencia insalvable entre la labor de Sánchez Sandoval y los preceptos estéticos fomentados por Caballero y Góngora, aunque podría tratarse del caso que ejemplifique un modus operandi promovido por la prelatura cordobesa destinado a integrar en la causa academicista a artífices de formación tradicional, con el fin de aprovechar sus capacidades técnicas.

\section{Arquitecto vs alarife. Debate edificatorio y controversia presupuestaria}

A pesar de todo, la edificación de la torre no estuvo exenta de dificultades. Pues, con motivo de las constantes advertencias expuestas a partir del 24 de marzo de 1792 por el maestro de obras Mariano Luque sobre la inseguridad de la obra ya ejecutada según el proyecto de Ignacio Tomás y los peligros que conllevaría su continuación, Navarro Villodres decretó el 14 de noviembre la paralización de las labores constructivas, así como el reconocimiento de la fábrica existente por un grupo de alarifes públicos. A propuesta del propio Tomás, fueron nombrados para llevar a cabo dicho cometido Alonso Torres, Vicente López Cardera, Cayetano Torres, Juan López Cardera y Francisco Jerez. El 19 de no- viembre se efectuó el reconocimiento, tras el cual los alarifes mencionados valoraron positivamente tanto el proyecto de Tomás como la labor de Luque, sin más recomendación que la de prestar atención en lo sucesivo a la solidez de la obra²2.

Poco tiempo después de la resolución del conflicto, en abril de 1793, Mariano Luque notificó a la autoridad diocesana la conclusión de la torre, a la que se habían añadido ciertos «aumentos y mejoras» pactados con Ignacio Tomás. En consecuencia, el día 24 de dicho mes, Navarro Villodres comisionó, para el reconocimiento de la obra ya finalizada, al arquitecto académico, quien aprobó el resultado y la labor del maestro alarife el 13 de mayo. Finalmente, entre el 15 y el 18 de junio se procedió a la liquidación de los gastos de la obra, que superaron en 9.349 reales de vellón y 17 maravedís los 60.000 reales de vellón estipulados en el presupuesto inicial, desglosados en: 3.904 reales de vellón y 31 maravedís, gastados de más por el asentista; y 5.444 reales de vellón y 20 maravedís, desembolsados desde el 31 de marzo hasta el 4 de julio de 1791 debido a un hundimiento de la fábrica. Dispendio extraordinario cuya primera partida no fue transigida por Navarro Villodres, quien exigió a Mariano Luque una verificación al respecto el día 19 de junio ${ }^{23}$.

Por consiguiente, Luque procedió a justificar los gastos no contemplados en el presupuesto de la obra, aportando para ello datos suplementarios sobre el proyecto ideado por Ignacio Tomás y las correcciones practicadas al mismo. Advirtiendo previamente que el diseño de la torre carecía de alzado y que para la ejecución del segundo cuerpo de la misma tuvo que seguir las indicaciones verbales del arquitecto episcopal, informó que el ancho de los pilares de dicho tramo pasaría de tres a cuatro pies (de 83 a $111 \mathrm{~cm}$ ), al igual que el ancho de los «ángulos» del primer cuerpo de 4 a 8 pies (111 a $222 \mathrm{~cm}$ ). Igualmente, en disconformidad con lo referido en el proyecto de Tomás, el citado cuerpo principal no se construyó de mampostería procedente de la fábrica primigenia y ladrillo, sino de cantería nueva, al igual que su imposta y las gradas del último cuerpo. Todo ello, más el gasto causado por la subida y asiento del escudo de armas del Obispo, la reforma de las cabezas de las campanas, así como la ejecución del cincho de hierro, la veleta y el nabo del remate, supondrían un dispendio de 8.534 reales de vellón por encima del presupuesto inicial. Unos 4.630 reales de vellón menos que los gastados realmente de más por el asentista. De este modo, Luque justificaba los gastos ex- 
traordinarios emprendidos y reivindicaba su quehacer como encargado de la obra, asegurando igualmente no solo que los cambios se habían emprendido conforme a lo acordado con Tomás, sino también que en la partida expuesta no se incluían los perjuicios sufridos durante los periodos en los que la obra se encontró paralizada ${ }^{24}$.

Una vez conocido lo expuesto por Luque, Navarro Villodres comisionó el 14 de julio a Tomás con el fin de que diera su parecer sobre el asunto referido y la cuantía que se le debía abonar por su trabajo como director de la obra, acerca de todo lo cual el arquitecto dictaminó un informe firmado el día 19 de dicho mes. Detalló en ocho puntos cada una de las partidas extraordinarias de la obra, las cuales sumarían a su entender un total de 3.618 reales de vellón. Igualmente, advirtió que los refuerzos de los ángulos y «otras piedras nuevas» se añadieron a la fábrica sin su consentimiento, por lo que no se le debía abonar nada a Luque en el desempeño de ese cometido. Por otra parte, indicó que sería de agradecer una «competente gratificación» económica por su trabajo, dados los cuidados y riesgos propios de la subida a los "palos y andamios hechos a la ligera» de la obra en cada uno de los reconocimientos diarios. En concreto, especificó que se le debían abonar a razón de 500 ducados anuales, la cantidad que según Tomás solían percibir los arquitectos académicos en Granada por el mismo trabajo, aun siendo la obra "de mucha menor consideración que la insinuada torre» 25 .

Tras conocer el informe de Tomás, Mariano Luque defendió por última vez su trabajo ante el Provisor mediante la presentación de un escrito centrado en denunciar el gran perjuicio que le había ocasionado la actitud del arquitecto académico, fundamentándolo en tres cuestiones principales. Comenzó destacando el enorme riesgo que habría causado para la solidez de la obra levantar el cuerpo principal de mampostería de la fábrica antigua y ladrillo (como sugería Tomás en su proyecto), atribuyéndose a sí mismo la acertada decisión de erigirlo enteramente de cantería. De igual modo, señaló que durante el transcurso del proceso constructivo había tenido que aguantar continuados «desazones, zozobras y riesgos", ya fuera por "ponerse en los mayores peligros» para fomentar la valentía de los obreros o por el mal trato recibido de Tomás, a quien acusó tanto de ausentarse continuadamente como de temer la subida a los andamios y evitarla constantemente. Por último, remarcó que el coste de todos los materiales, el andamiaje y la maquinaria desti-

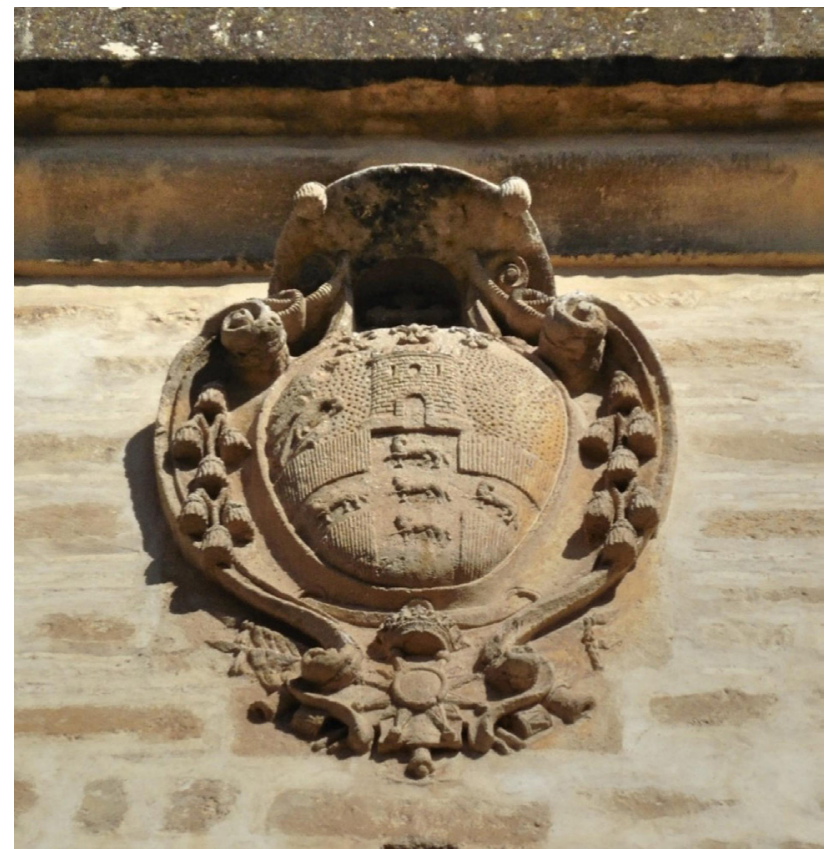

2. Manuel Sánchez Sandoval. Escudo de armas del obispo Caballero y Góngora de la torre de la iglesia de la Magdalena

nados a la construcción de la torre habían sido adquiridos a menor coste gracias a su gestión, tal y como había reflejado en su testimonio anterior. Razón por la cual entendía que las modificaciones del proyecto habían supuesto una mejora sustancial del mismo a muy bajo coste, a pesar de las contradicciones y desaprobaciones continuas de Tomás ${ }^{26}$.

Finalmente, «teniendo en cuenta todo lo referido en los memoriales de los aumentos», Navarro Villodres decretó el día 4 de septiembre la gratificación que cada uno de los implicados debía percibir. A Mariano Luque, «acreedor de una importante competencia y gratificación», estimó que se le eximiera del pago de los 3.904 reales de vellón y 31 maravedís gastados de más en la obra, así como que se le abonaran 100 doblones. Por el contrario, Ignacio Tomás recibió únicamente 50 doblones, en atención a una falta de continuidad en su asistencia a la obra y a la "decente dotación» de mil ducados anuales que recibía de la mitra cordobesa (Ponz, 1792: 38)²7.

Paradójicamente, los consabidos beneficios del cargo que Tomás ocupaba al servicio de la prelatura cordobesa le perjudicaron en la liquidación de la obra, la cual, si bien se finalizó siguiendo los parámetros formales propuestos por aquel, no se construyó con los materiales especifica- 


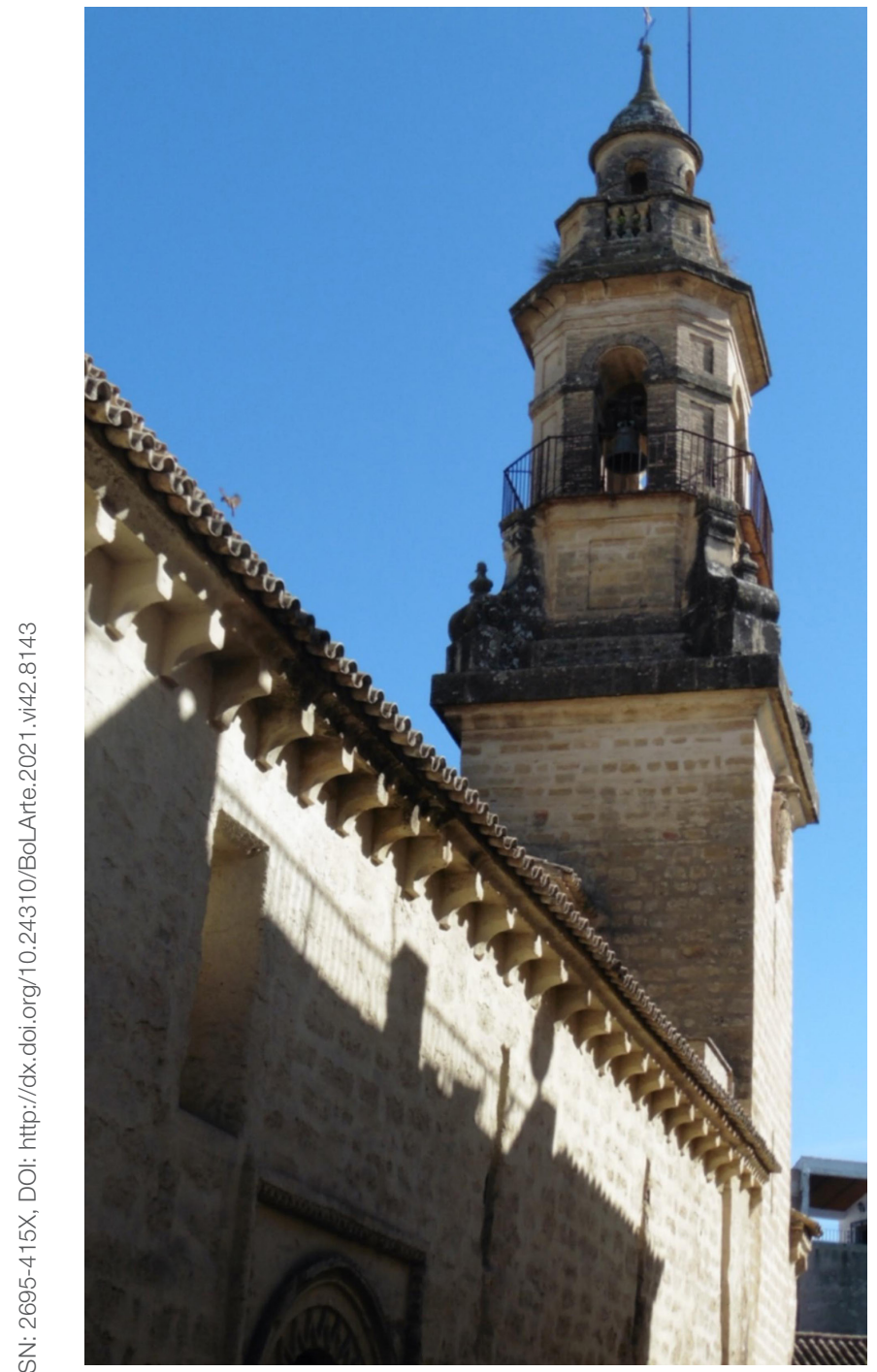

3. Torre de la iglesia de Santa María Magdalena de Córdoba

dos en su proyecto inicial. Esta no fue la primera ocasión en la que el arquitecto académico veía modificado alguno de sus proyectos como consecuencia de la opinión contraria de un maestro alarife de formación tradicional. Un año antes, Manuel Núñez estimó como inapropiados los materiales propuestos por Tomás para la reforma y terminación de la parroquia mayor de Santa Cruz de Écija. Lo cual persuadió al Arzobispado hispalense de dictaminar que la obra se emprendiese siguiendo el diseño aportado por el arquitecto académico, mas con las modificaciones estructurales indicadas por el alarife sevillano (Ruiz, 2019: 44-46). Aunque en

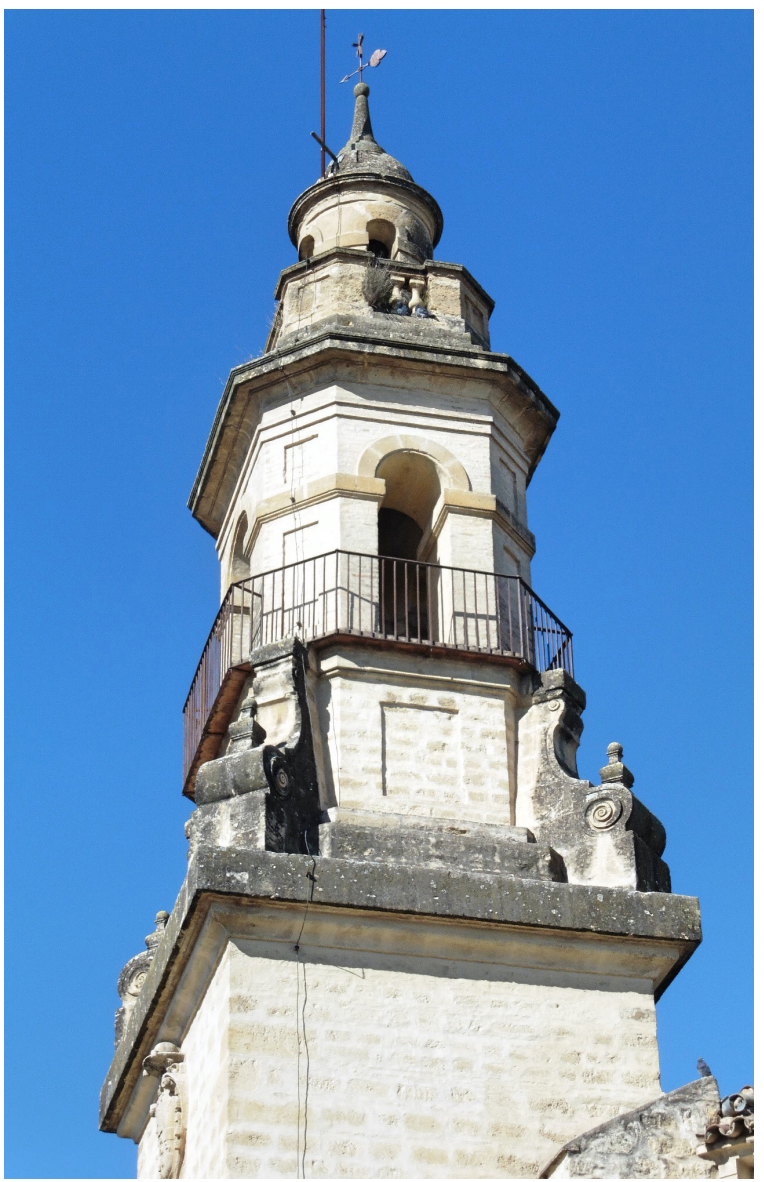

4. Vista de los dos cuerpos superiores y del remate de la torre de la iglesia de Santa María Magdalena de Córdoba

la referida ocasión Tomás denunció sin éxito la intervención de Núñez ante la Corona, al encontrarse en una posición considerablemente más desfavorable y como consecuencia de su conocido carácter reivindicativo ${ }^{28}$, en ambas situaciones los motivos por los que los alarifes reprobaron sendos proyectos fue el mismo: la elección de mezclas de ladrillo o mampostería destinadas a conformar los elementos sustentantes de las obras, en detrimento de la piedra. Dicha cuestión resulta fundamental para comprobar la importancia que los arquitectos académicos otorgaban al abaratamiento de los costes de las obras, pues tenían especialmente asumidos los problemas económicos del Reino. Del mismo modo que evidencia una nueva cuestión sobre el conflicto con los alarifes tradicionales, que no solo se opusieron a las nuevas directrices formales, sino también, a los materiales. 


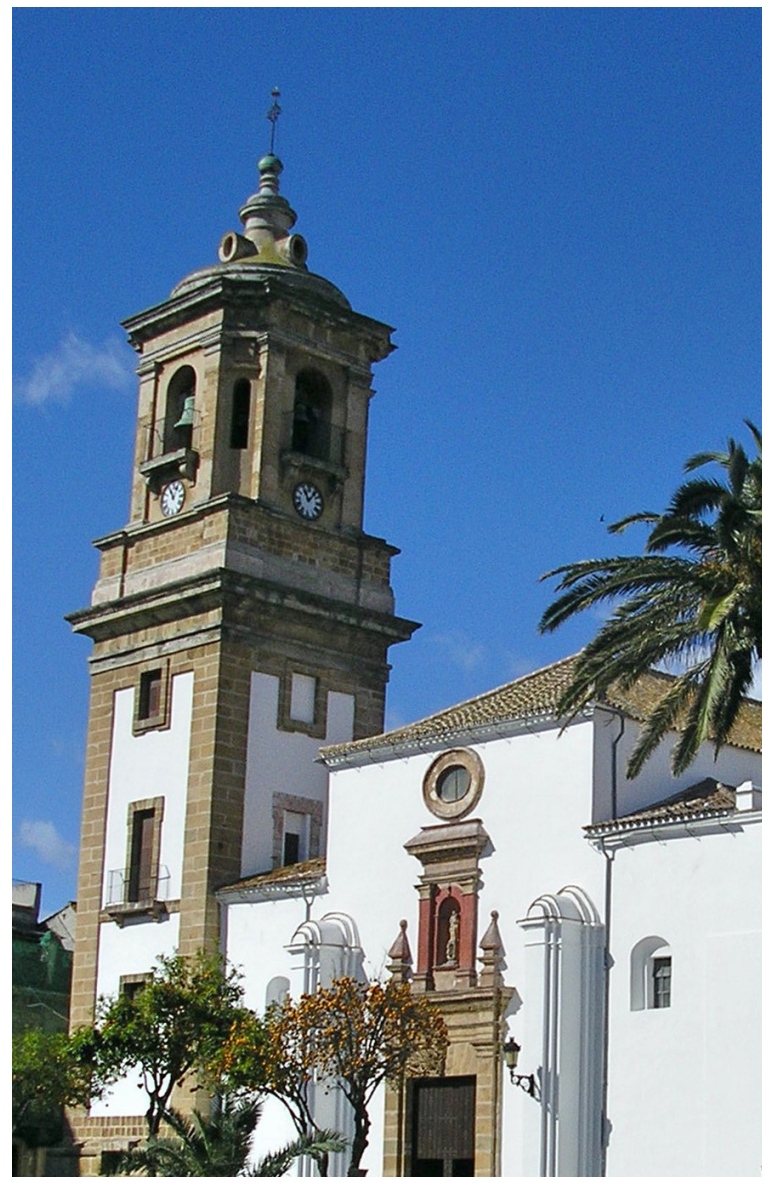

5. Manuel Machuca Vargas. Torre de la parroquia de Nuestra Señora de la Palma de Algeciras

\section{Consideraciones estéticas y conclusivas}

A pesar de todo, el resultado final de la obra corresponde con la idea planteada por Ignacio Tomás, de estética academicista [3]. Sustentada sobre el gran cuerpo principal de base cuadrada, en cuya parte superior del lado sur se ubica el escudo de armas del obispo Caballero y Góngora, la torre de la iglesia de la Magdalena destaca formalmente por sus dos cuerpos superiores ochavados, rematados con un chapitel cónico cercado por una balaustrada que mantiene los chaflanes correspondientemente [4]. Esta configuración, destinada a atenuar «la dureza y aspereza de la forma cuadrada» (Bails, 1796: 847-848), es prototípica de la arquitectura academicista, como se puede comprobar en otros ejemplos contemporáneos del mismo

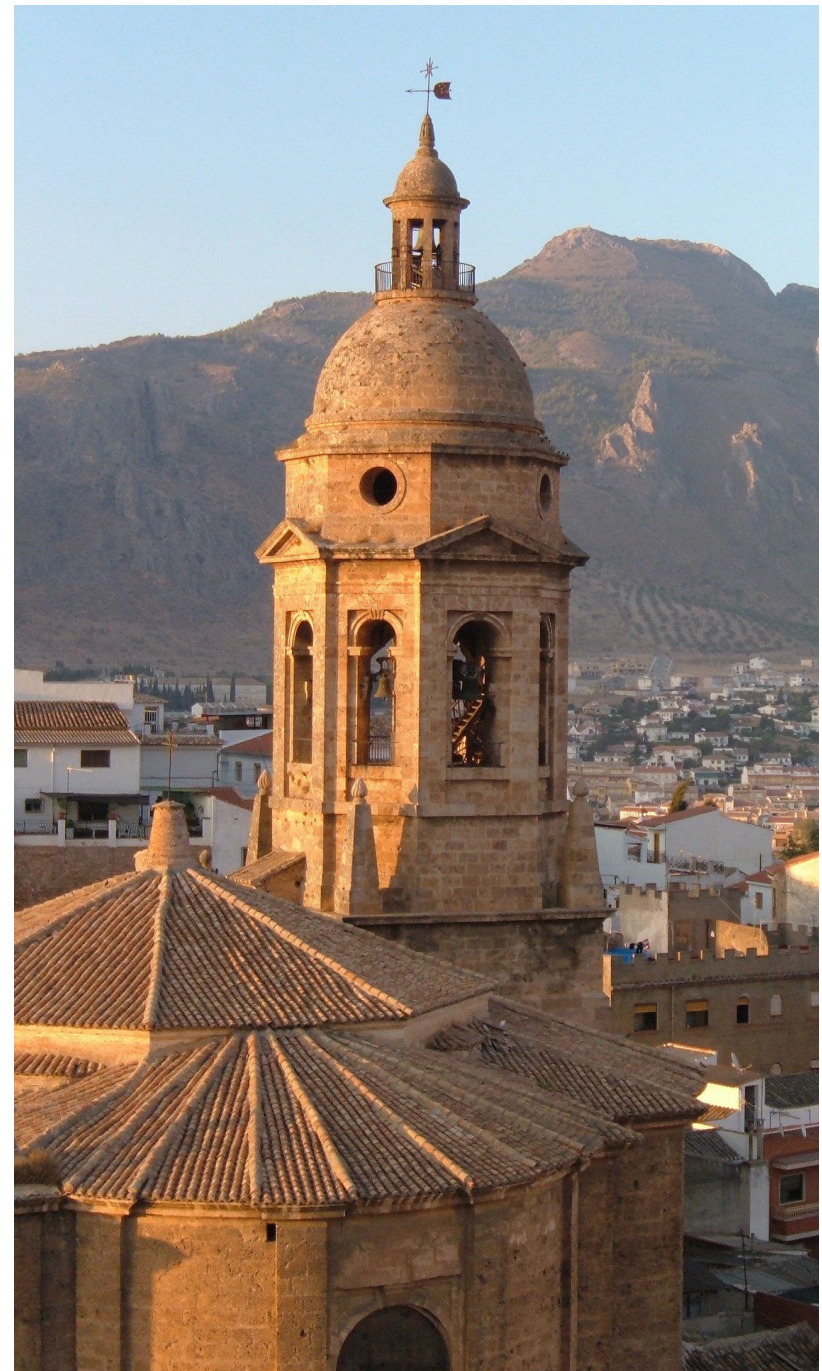

6. Ventura Rodríguez y Domingo Tomás. Torre de la parroquia mayor de Santa María de la Encarnación de Loja

género proyectados por artistas formados en la Academia madrileña junto al propio Tomás. Tal es el caso de la torre de la parroquia de Nuestra Señora de la Palma de Algeciras, diseñada por Manuel Machuca Vargas en 1791 [5]; o aquella correspondiente a la parroquia mayor de Santa María de la Encarnación de Loja, trazada por Domingo Tomás (hermano de Ignacio) en 1797 siguiendo una idea inicial de Ventura Rodríguez ${ }^{29}$ [6]. No obstante, en la torre cordobesa se incorpora una variante original que la diferencia de las mencionadas y de otros ejemplos academicistas: la presencia de aletones con volutas situados en el segundo 


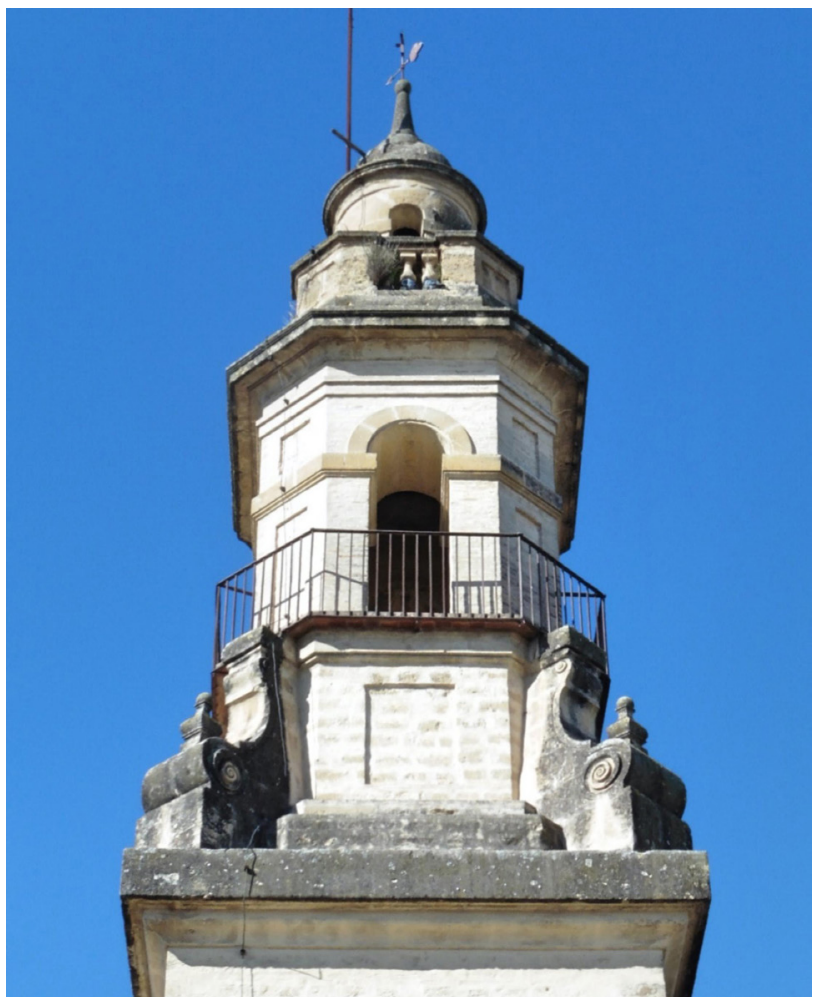

cuerpo [7]. Elementos que no solo le otorgan una mayor plasticidad al conjunto, sino que también marcan elocuentemente la transición entre la base cuadrangular del primer cuerpo y la ochavada del tercero.

La edificación de la torre de la iglesia de la Magdalena representa los diferentes parámetros de la introducción de los preceptos arquitectónicos academicistas en la Diócesis de Córdoba. Proceso que, aun siendo patrocinado oficialmente por el prelado Caballero y Góngora, contó con dificultades en su puesta en marcha, tanto por los errores implícitos en el afán académico, como por los cambios que significaba para el ejercicio de la disciplina constructiva en lo formal y en lo profesional. Mas la decidida voluntad institucional por desarrollar la iniciativa significó el inicio de la evolución del arte cordobés hacia la contemporaneidad. Un punto de inflexión en su historia.

\section{Lado oriental de los cuerpos superiores y remate de la torre de la iglesia de Santa María Magdalena de Córdoba}

\section{Notas}

1 Si bien la ciudad cordobesa contó en las décadas de 1760 y 1770 con la presencia de Miguel Verdiguier, Luis Gilbert y José Dréveton, que llevaron a cabo obras estéticamente acordes con el gusto internacional del momento (Rivas, 1986); y teniendo en cuenta las empresas artísticas promocionadas por el VI conde de Fernán Núñez en su localidad originaria (Vigara, 2014; 2017), no vinculadas con la Academia pero cercanas formalmente a los preceptos que ésta promulgaba; la única intervención académica en Córdoba hasta el año 1790 fue la protagonizada por Ventura Rodríguez en la iglesia del colegio de Santa Victoria en 1772 (Pérez, 2005: 313-319)

2 Quien, concretamente, arribó a la ciudad el 19 de diciembre de 1789. Archivo de la Catedral de Córdoba, Córdoba (ACC), Actas Capitulares, 92, ff. $101 \mathrm{v}-104 \mathrm{v}$.

3 Todos ellos reconocidos como «académicos de mérito»: Ignacio Tomás en 1774, Joaquín Arali en 1780 y Francisco Agustín, que cuando llegó a Córdoba era un alumno de la Academia pensionado en Roma, en 1792 (Real Academia de San Fernando, 1793: 128-134).

4 Archivo de la Real Academia de Bellas Artes de San Fernando, Madrid (ARABASF), Secretario general, Libro de actas de la Comisión de Arquitectura, 3-139, ff. $135 \mathrm{v}-136 \mathrm{r}$.

5 Archivo General del Obispado de Córdoba, Córdoba (AGOC), Autos ordinarios, 8205, exp. 21, s/f.

6 ACC, Cuentas de Fábrica, 4042, s/f.

7 A partir del incendio acontecido en mayo de 1872 (Ramírez de Arellano, 1873: 11), el templo padeció un largo y progresivo deterioro que causó la pérdida de su condición como parroquia en 1890 y, posteriormente, su desacralización en 1956. Desde entonces, la primitiva iglesia fue abandonada, así como paulatinamente saqueada en su mayoría; hasta que otro incendio sobrevenido el 5 de septiembre de 1990 acarreó la destrucción de sus cubiertas y de parte de las obras lignarias aún existentes. Lo que quedó del conjunto fue remodelado entre los años 1995 y 1998. Haba, 2016.

8 Se trata de la parroquia correspondiente a una de las catorce collaciones fundadas por Fernando III tras la conquista de Córdoba en 1236. Igualmente, se cree que la construcción del templo primigenio comenzó en la segunda mitad del siglo XIII. Jordano, 2002: 27-58.

9 «Por cada diferente diminucion, ò por cada alto conviene mucho, que las paredes puestas se unan, y traben con tirantes, para que tanto mas firmemente resistan à la opresion del peso, que se les impone». Benavente, 1763: 60-61.

10 AGOC, Despachos ordinarios, 7132, exp. 41, ff. 1r-3r.

11 AGOC, Despachos ordinarios, 7132 , exp. 41, ff. 4r-5v.

12 Sobre las mezclas de cal y arena véase Villanueva, 1827: 13-16.

13 AGOC, Despachos ordinarios, 7132, exp. 41, f. 4r. 
14 Véanse las diferentes formas de paramentar los muros de los edificios, directamente relacionadas con lo expuesto por Tomás, contenidas en Ortiz y Sanz, 1787: 42-49.

15 Conocido también como "goterion» o "goteron", puede definirse como: «Canal que se hace en las coronas en la parte de abaxo, con el fin de que caygan al suelo gota á gota las aguas llovedizas que caen encima de la cornisa; sin cuya precaucion se escurririan hasta el sofito ó cielo raso de la corona». Bails, 1802: 51.

16 AGOC, Despachos ordinarios, 7132, exp. 41, ff. 4r-5r.

17 AGOC, Despachos ordinarios, 7132, exp. 41, ff. 5r-5v.

18 Quien también ostentaba los cargos de consultor del Santo Oficio e inquisidor honorario del Cabildo de la Catedral de Córdoba. Rey, $1924: 127$.

19 Lo cual suponía un aumento con respecto a la cantidad valorada por Tomás. Si bien cabe reseñar que Mariano Luque solicitó que la obra debía tasarse en 62.000 reales de vellón y que los gastos que excedieran del presupuesto inicial no recayeran en su persona. Ambas demandas fueron desestimadas. AGOC, Despachos ordinarios, 7132, exp. 41, ff. 5v-6r, 24r-28r.

20 AGOC, Despachos ordinarios, 7132, exp. 41, ff. 39v-41v, 57r.

21 Los datos presentados, además, revelarían la última obra conocida realizada por Sánchez Sandoval (Valverde, 1974: 293-296; Raya, 1987: 22, 188, 194, 224, 300, 301; García, 1997: 182-186).

22 El reconocimiento, cuyo coste total ascendió hasta los 632 reales de vellón, se llevó a cabo en presencia de Tomás, Luque, el rector de la parroquia, su sacristán mayor y el notario mayor de gobierno, Miguel Toledano Alfonso. AGOC, Despachos ordinarios, 7132, exp. 41, ff. 64r-67r.

23 En contraposición con la segunda partida, que fue comprendida por su carácter accidental. AGOC, Despachos ordinarios, 7132, exp. 41, s/f.

24 AGOC, Despachos ordinarios, 7132, exp. 41, s/f.

25 AGOC, Despachos ordinarios, 7132, exp. 41, s/f.

26 AGOC, Despachos ordinarios, 7132, exp. 41, s/f.

27 AGOC, Despachos ordinarios, 7132, exp. 41, s/f.

28 ARABASF, Comisión de Arquitectura, Informes, Iglesias parroquiales, 2-33-2, exp. 1, s/f.

29 ARABASF, Secretario general, Libro de actas de la Comisión de Arquitectura, 3-139, ff. 171r y $295 \mathrm{v}$.

\section{Bibliografía}

ARANDA DONCEL, Juan (1986), «Un proyecto ilustrado en la Córdoba del siglo XVIII: La Escuela de Bellas Artes del obispo Caballero y Góngora», Aphoteca, n. ${ }^{\circ}$, pp. 33-49.

BAILS, Benito (1796), Elementos de Matemática. Tomo IX. Parte I. Que trata de la Arquitectura Civil, Imprenta de la Viuda de D. Joaquín Ibarra, Madrid.

BAILS, Benito (1802), Diccionario de Arquitectura Civil, Imprenta de la Viuda de Ibarra, Madrid.

BÉDAT, Claude (1989), La Real Academia de Bellas Artes de San Fernando (1744-1808), Fundación Universitaria Española-Real Academia de Bellas Artes de San Fernando, Madrid.

BENAVENTE, Miguel (1763), Elementos de toda la architectura civil, con las mas singulares observaciones de los modernos impresos en latín por el P. Christiano Rieger de la Compañía de Jesús, al presente Cosmographo Mayor de S. M. y de su Consejo en el Real y Supremo de Indias, Maestro de Matemáticas del Colegio Imperial, Joachin Ibarra, Madrid.

GARCÍA DE LA TORRE, Fuensanta (1997), Dibujos del Museo de Bellas Artes de Córdoba, Junta de Andalucía, Sevilla.

GARCÍA MELERO, José Enrique (1991), «Arquitectura y burocracia: el proceso del proyecto en la Comisión de Arquitectura de la Academia (1786-1808)», Espacio, tiempo y forma. Serie VII, Historia del arte, n. ${ }^{\circ} 4$, pp. 283-384.

HABA, Rafael de la (2016), «El Incendio de la Magdalena», Diario Córdoba, 6 de septiembre, p. 19.

JORDANO BARBUDO, María Ángeles (2002), Arquitectura medieval cristiana de Córdoba, T. II, tesis doctoral inédita, Universidad Complutense de Madrid, Madrid.

ORTIZ Y SANZ, Joseph (1787), Los Diez Libros de Architectura de M. Vitruvio Polión, Imprenta Real, Madrid.

PÉREZ AYALA, José Manuel (1951), Antonio Caballero y Góngora. Virrey y Arzobispo de Santa Fe. 1723-1796, Imprenta Municipal, Bogotá.

PÉREZ MARÍN, María Dolores (2005), Escolapias en Andalucía. Universidad de Córdoba-Cajasur, Córdoba.

PONZ, Antonio (1792), Viage de España, T. XVII, Imprenta de la Viuda de D. Joaquin Ibarra, Madrid.

RAMÍREZ DE ARELLANO Y GUTIÉRREZ, Teodomiro (1873), Paseos por Córdoba, T. I, Imprenta de D. Rafael Arroyo, Córdoba.

RAYA RAYA, María Ángeles (1987), Retablo Barroco Cordobés, Publicaciones del Monte de Piedad y Caja de Ahorros de Córdoba, Córdoba. 
REAL ACADEMIA DE SAN FERNANDO (1790), Distribución de los premios concedidos por el rey nuestro señor á los discípulos de las nobles artes, hecha por la Real Academia de San Fernando en la Junta pública de 4 de Agosto de 1790, Imprenta de la Viuda de Ibarra, Madrid.

REAL ACADEMIA DE SAN FERNANDO (1793), Distribución de los premios concedidos por el rey nuestro señor à los discípulos de las tres nobles artes, hecha por la Real Academia de San Fernando en la Junta pública de 20 de agosto de 1793, Imprenta de la Viuda de Ibarra, Madrid.

REY DÍAZ, José María (1923), «D. Antonio Caballero y Góngora. Arzobispo-Virrey de Nueva Granada (III)», Boletín de la Real Academia de Ciencias, Bellas Artes y Nobles Artes de Córdoba, n. ${ }^{\circ}$ 6, octubre-diciembre, pp. 57-76.

REY DÍAZ, José María (1924), «D. Antonio Caballero y Góngora. Arzobispo-Virrey de Nueva Granada (Conclusión)», Boletín de la Real Academia de Ciencias, Bellas Artes y Nobles Artes de Córdoba, n. ${ }^{\circ}$, enero-marzo, pp. 101-113.

RIVAS CARMONA, Jesús (1986), «Notas para el Neoclásico cordobés», Imafronte, n. 2, pp. 25-55.

RUIZ CARRASCO, Jesús María (2019), «La labor del académico Ignacio Tomás en Écija, su disputa con los arquitectos sevillanos y la construcción de la parroquia mayor de Santa Cruz», Archivo Español de Arte, vol. 92, n. ${ }^{\circ}$ 365, pp. 37-50.

SAMBRICIO, Carlos (1986), La Arquitectura española de la llustración, Consejo Superior de los Colegios de arquitectos de España- Instituto de Estudios de la Administración Local, Madrid.

SERRA MASDEU, Anna Isabel (2014), «La biblioteca del arquitecto y académico Ignasi Tomàs Fabregat (c. 1744-1812)», Cuadernos de Arte de la Universidad de Granada, n. ${ }^{\circ}$ 45, pp. 129-141.

VALVERDE MADRID, José (1974), Ensayo socio-histórico de retablistas cordobeses del siglo XVIII, Publicaciones del Monte de Piedad y Caja de Ahorros de Córdoba, Córdoba.

VIGARA ZAFRA, José Antonio (2014), «El palacio del VI conde de Fernán Núñez: la arquitectura como exaltación simbólica del linaje durante la llustración», Tiempos modernos: Revista Electrónica de Historia Moderna, vol. 8, n. ํ29, s/f.

VIGARA ZAFRA, José Antonio (2017), «Nobleza versus Clero: el patronato de la Iglesia de Santa Marina en Fernán Núñez durante la Edad Moderna", Revista de Humanidades, n. ${ }^{\circ}$ 30, pp. 75-94.

VILLANUEVA, Juan (1827), Arte de Albañilería, Oficina de Don Francisco Martínez Dávila, Madrid. 